\title{
PERSONAL CONSTITUTION AND HEALTH STATUS AMONG CHINESE ELDERLY IN TAIPEI AND LOS ANGELES
}

\author{
CHIH-YIN LEW-TING, ${ }^{1 *}$ MARGO-LEA HURWICZ ${ }^{2}$ and EMIL BERKANOVIC ${ }^{3}$ \\ ${ }^{1}$ School of Public Health, National Taiwan University, No. 1, Sec. 1 Jen-Ai Road, Taipei, Taiwan, \\ R.O.C., ${ }^{2}$ Department of Anthropology and Gerontology Program, University of Missouri, St. Louis, \\ MO, U.S.A. and ${ }^{3}$ Department of Community Health Sciences, School of Public Health, University of \\ California, Los Angeles, CA, U.S.A.
}

\begin{abstract}
This paper reports ethnographic and statistical data on the indigenous Chinese concept of constitution (ti-zhi) and its association with health status. The data were obtained through in-depth interviews with 203 Chinese elderly residing in Taipei and Los Angeles. The informants used several dimensions to characterize their constitutions. They also described how their constitutions originated and were expressed over the lifecourse. The association between these characterizations of constitution and reported number of symptoms/diseases, self-rated health status and satisfaction with health were examined statistically. These analyses indicate that characterizations of constitution as strong/weak or as hot/cold, have significant but moderate associations with the indicators of health status. (C) 1998 Elsevier Science Ltd. All rights reserved
\end{abstract}

Key words - Chinese elderly, personal constitution, health status

\section{INTRODUCTION}

Health status is a key construct in Western research on how people assess their physical well being. Used both as an independent variable (e.g., as a predictor of various health and illness behaviors) and as a dependent variable (e.g., as an outcome of sociocultural, economic, residential and other conditions), health status is, perhaps, one of the most widely examined constructs in gerontological health research.

Although the concept of health status has been quite useful in Western analyses of conceptions of health (Bergner, 1987), it is a culture bound term that reflects the views of the Western medical establishment regarding the diagnosis, treatment, and probable cure of disease. It may not be as useful in understanding people from other cultures, who may have other models to explain their own physical well being. For example, Chinese concepts of health may be better understood within the context of Chinese medical traditions and local health beliefs.

In this study, we examine the Chinese concept of constitution as an indigenous construct that serves as part of an explanatory model for understanding

*Author for correspondence. a number of aspects of life, including physical well being. We will show how contemporary elderly Chinese describe this construct as a pervasive influence on their health and other aspects of their life experiences. In addition to the attributes of their personal constitution, they explain its origins and expression over the life course. By presenting the locutions and phrases used by elderly Chinese to characterize their constitution, this study takes an emic approach. Using statistical analyses, it further explores the relationship between the culturally derived dimensions of this construct and the responses of Chinese elders to Western survey questions about health status.

\section{CONSTITUTION IN CHINESE CULTURE}

Constitution (ti-zhi) is a widely used term in Taiwan. Literally, $t i$ means body and $z h i$ denotes quality or substance. In Chinese medicine, personal constitution is an important criterion for diagnosis and treatment ( $\mathrm{Su}, 1992)$. Constitution and the classes into which it can be categorized was first documented in the classic Chinese medical text Nei-Jing (Inner Classic of the Yellow Sovereign) (Lu, 1982). Subsequent Chinese medical studies of constitution were based on the tradition established by this text. The Nei-Jing 
provides three axes on which personal constitution is classified: (1) morphological and physical features; (2) the yin-yang quadrant*; and (3) the five evolutive phases (wu-xing) $\dagger$. Thus, the classification of constitution in the Nei-Jing is characterized by a comprehensive line of theory that includes physical, personality, and behavioral manifestations. Clinically, personal constitution in traditional Chinese medicine can be viewed as an individual's susceptibility or vulnerability to specific diseases or symptoms ( $\mathrm{Su}, 1992)$.

Physical constitution as a medical concept has been explored using Chinese medical classics ( $\mathrm{Li}$, 1981; Mu, 1983; Shi, 1984). More recently it has also been examined in relation to a specific disease category ( $\mathrm{Su}, 1992)$, the distribution of a genetic trait (Zhou, 1987), the biochemistry of dietary items (Zhang, 1992), and modern immunologic theory (Tian, 1984). Despite enthusiasm among modern Chinese scholars about the clinical implications of constitution (Wang, 1989), the study of the lay concept of constitution from an emic perspective has, to our knowledge, not been addressed $\$$.

While a taxonomy of constitution has been extensively elaborated in Chinese medicine, attributions related to personal constitution are also commonly used by lay people. While these lay ideas about constitution may not correspond fully to the understanding developed by classical Chinese medicine, they are important because they both affect how people think about their health and what they do about it.

The present study is concerned with what older Chinese actually say about their personal constitution. What they say about their constitutions and how they say it may give us insight into what they believe about the relationship between their constitutions and their health. Two dimensions of personal constitution coded from their open-ended responses will be compared to their responses to standard Western survey questions about health status to explore the extent to which

*Yin-yang as a dimension to classify personal constitution can be further divided into: minor yin (shao-yin), major yin (tai-yin), minor yang (shao-yang), major yang (tai-yang), and harmonious yin-yang (yin yang tiao he) (Su, 1992).

$\uparrow$ Five evolutive phases is a theory of change and movement in Chinese medicine, as the word "xing" implies. Wood, fire, metal, and water characterize four critical phases in any evolution. Earth represents neutral indifferentiation, or the transitions between phases of specific quality. For a brief review of this theory, see Porkert (1977).

\#Although the concept of constitution is mentioned in passing in anthropological studies involving traditional Chinese medicine (Sankar, 1984; Furth and Ch'en, 1992), we know of no anthropological study focused exclusively on constitution $(t i-z h i)$. the Chinese construct and the Western construct are related.

\section{THE RESEARCH SETTINGS}

Data for this exploratory investigation were drawn from a comparative study on self healthpromotive behavior of elderly Chinese residing in retirement homes in two cities. The four retirement homes chosen for this study, two in Los Angeles, California, U.S.A. and two in Taipei, Republic of China, are non-profit, public housing projects established in the early 1980s.

The two communities in Los Angeles represent different geographic settings and socio-demographic compositions. Angeles Plaza, located in the western portion of downtown Los Angeles, is characterized by its multicultural and multilingual population. About one-fourth of the residents are ethnically Asian, of whom approximately 250 are Chinese. The majority of these are immigrants from Taiwan. Golden Age Village is situated in a lower middle class suburban neighborhood that has a considerable number of new Chinese immigrants. More than $90 \%$ of the residents of this home are Chinese, predominantly immigrants from Taiwan.

Evergreen Village and Cypress Home, the communities in Taiwan, were the first two residential retirement facilities for senior citizens in Taipei City. Situated in a commercial-residential neighborhood in a northern suburb of Taipei, Cypress Home has a unique ambiance. It is a community where residents can enjoy a pleasing environment of quiet and fresh air, while taking advantage of their proximity to resources and amenities provided by Taipei City. Perched on the slopes of Mount Wu-chi in suburban Taipei, Evergreen Village is the most geographically isolated of the four research communities. Nevertheless amenities have been developed to make it a somewhat selfsustaining community where the elderly can enjoy a life of seclusion.

\section{METHOD}

In addition to participant observation in the four communities, including informal interviews with community and staff members, face-to-face interviews were conducted using a semi-structured questionnaire. Based on a stratified sampling scheme designed to represent the four communities, a total of 203 elderly residents were selected and interviewed by the senior author between December 1990 and July 1991.

\section{The informants}

As shown in Table 1, the Taipei residents $(n=89)$ and the Los Angeles residents $(n=114)$ resemble each other in many ways. Both samples 
Table 1. Comparison of sociodemographic characteristics and type of constitution of two study groups

\begin{tabular}{|c|c|c|c|}
\hline & Los Angeles $(n=114)(\%)$ & Taipei $(n=89)(\%)$ & Total $(N=203)(\%)$ \\
\hline Sex & & & (n.s) \\
\hline Male & 49.1 & 47.2 & 48.3 \\
\hline Female & 50.9 & 52.8 & 51.7 \\
\hline Age & & & (n.s) \\
\hline $59-70$ & 19.3 & 5.6 & 13.3 \\
\hline $71-80$ & 64.0 & 64.0 & 64.0 \\
\hline $81-90$ & 16.7 & 30.3 & 22.7 \\
\hline Mean years of age & 75.4 & 77.6 & 76.4 \\
\hline Education & & & (n.s.) \\
\hline Junior high and below & 22.8 & 23.6 & 23.2 \\
\hline High school & 31.6 & 42.7 & 36.5 \\
\hline College and above & 45.6 & 33.7 & 40.4 \\
\hline Mean years of education & 12.6 & 11.7 & 12.2 \\
\hline \multicolumn{4}{|l|}{ Constitution (ti-zhi) } \\
\hline Strong/normal/weak dimension & & & (n.s.) \\
\hline Strong & 41.2 & 23.6 & 33.5 \\
\hline Normal & 30.7 & 36.0 & 33.0 \\
\hline Weak & 11.4 & 12.7 & 9.8 \\
\hline Hot/cold dimension & & & (n.s.) \\
\hline Hot & 10.5 & 12.4 & 11.3 \\
\hline Cold & 6.1 & 11.2 & 8.4 \\
\hline
\end{tabular}

are fairly evenly divided between men and women. The elders from Los Angeles are both younger and better educated than the elders from Taipei; however these differences are not significant statistically. With a single exception, the informants in both groups were born in mainland China and have shared experiences during the same specific historical eras. The members of the Los Angeles group are all immigrants from Taiwan. The length of their residence in the United States ranges from 4 to 23 years, with an average of 12 years. The apparent difference in the distribution by group of the $t i-z h i$ responses also is not statistically significant. What is more important is that both groups used the same set of categories to answer the question about constitution.

\section{The interview}

The average length of interview was $75 \mathrm{~min}$, varying from a minimum of $45 \mathrm{~min}$ to a maximum of more than $4 \mathrm{~h}$. Questions concerning personal constitution encompassed not only the informant's assessments of their own constitutions, but also their beliefs regarding the cognitive domain of personal constitution and its role in health maintenance. The elders were not asked to define constitution. They were asked to indicate the characteristics of their own constitution: "What would you say about your own constitution ( $N i$ jue de ni de ti zhi ru he)?" All responded with no difficulty, indicating that the concept of constitution is part of their everyday store of knowledge from which they can draw with ease.

Three standard, closed-ended, survey questions were asked: (1) Would you say your health status is very good, good, fair, poor, or very poor ( $D a t i$ shuo lai, nin ren wei nin de jian kang zhuang kuang ru he? fei chang hao, hao, pu tong, bu hao, fei chang bu hao)? (2) In general, how satisfied would you say you are with your health status? Are you very satisfied, satisfied, fairly satisfied, dissatisfied or very dissatisfied (Zong he lai shuo, nin dui mu qian de jian kang zhuang kuang gan dao man yi ma? fei chang man yi, man yi, pu tong, bu man yi, fei chang bu man yi)? (3) I am going to read you a list of symptoms or diseases people of your age may have. Do you have any of these health problems? (A pin yin translation of the list is available from authors on request).

\section{The analysis}

The open-ended responses obtained in the interviews illustrate the Chinese conceptual dimensions underlying personal constitution. The verbatim comments of the elderly Chinese are presented and analyzed. The purpose of this analysis is to reveal general trends as much as to demonstrate significance (Gephart, 1988). Then the statistical relationships of the respondents' global ratings of their own constitution to their responses to standard Western survey questions on health status and satisfaction with personal health are examined. Since there are no statistically significant differences between the Taipei residents and the Los Angeles residents in either the demographic or the constitution variables, the two groups are pooled for analysis.

\section{HOW CONSTITUTION IS DESCRIBED}

As presented in Table 2, positive, neutral and negative expressions were the most common descriptors of personal constitution. Terms such as "good", "excellent", "bad", or "general" were used by $78 \%$ of the informants to describe the overall 
Table 2. Locutions used to characterize personal constitution

\begin{tabular}{lr}
\hline & $N(\%)$ \\
\hline - Strong/weak or good/bad & $159(78.3)$ \\
- Tenacious, flexible, resistant, strong, high & $68(33.5)$ \\
quality, very good, good, not bad, etc. & $71(35.0)$ \\
- Normal, not special, the same as others, etc. & $20(09.9)$ \\
- Bad, weak, etc. & $47(23.2)$ \\
Hot/cold & $23(11.3)$ \\
- Hot & $17(08.4)$ \\
- Cold & $7(03.4)$ \\
- Sometimes hot and sometimes cold & $24(11.8)$ \\
Other dimensions & $7(03.4)$ \\
- Skinny, fat, etc. & $7(03.4)$ \\
- Good temper, enduring, patient, easy going, & \\
easy to get nervous, etc. & $6(03.0)$ \\
- Low blood pressure, B blood type, low & \\
temperature, early menstruation & $4(02.0)$ \\
- Intelligent, bright &
\end{tabular}

Note: Some informants mentioned more than one dimension so that percentages do not sum to 100 .

quality of their constitution. About one-third of the sample $(35 \%)$ felt the quality of their constitution was neutral, using words like "ordinary", "normal", and "nothing special", to describe it. They considered theirs "was just the same as other people's constitutions". Almost as many (34\%) held a very positive view of their constitution and used words such as "strong", "enduring", "flexible", "resistant", or "tenacious" to characterize it. Ten percent of the sample stated that their constitution was "bad" or "weak", and $22 \%$ did not use a descriptor from this dimension to characterize their constitution.

In addition to the overall descriptions that characterized the quality of their constitutions, evidence was further provided by most of the elderly to support their viewpoint. Mr. Cheng, for example, stressed that he had a strong, tenacious constitution because he could "take what comes", an ability that was the result of his experience with adverse environments during his childhood. Similarly, Mrs. $\mathrm{Wu}$ was very satisfied with her constitution saying she could "feel happy everyday and never get tired". A number of respondents felt proud of their constitution for they "had no fear of cold". They said they believed that a strong constitution enabled a person to stand not only cold weather but also other unfavorable conditions.

Some people emphasized the influence of mental processes on their constitutions. In talking about his "super good" constitution, the 78-yearold Mr. Yuang proudly remarked:

One's constitution has a lot to do with health and intelligence. Since I have got an excellent genetic heredity

*This is related to, but should not be confused with humoral medicine. See Foster (1987) for a discussion of how a formal ethnomedical system can become a folk tradition through diffusion to nonliterate populations. from my parents, my children and grandchildren are all Ph.D.s.

The most common manifestation of good constitution, as indicated by the elders, was freedom from physical disorders. They frequently used the following kind of statement to justify their superior constitution: "rarely get sick", "no pain and disease", "no cardiovascular diseases", "no diabetes", "no big health problems except having a minor surgery 3 years ago", or "never visit a hospital before". One respondent described her constitution:

In spite of little sleep and little food I am still very energetic. What I need is very little. I usually wear very few clothes but I can endure either cold or hot conditions.

Another group of people took an explicitly comparative stance in describing their constitution. They considered their constitutions "better than other people", "better than people of my age", "better than at least $70 \%$ of people the same age as mine". Some, however, related their constitution to age. They said that without a good constitution how can one live so long. An 80-year-old stressed, "I think I have a good constitution because I feel I am just as young as 50 ".

Poor functional performance and health status constituted the primary reason leading people to give negative estimates of their constitution, such as "easy to get sick", and "used to be weak and feeble; cannot move heavy stuff". Some, like Mrs. Liang provided more specific justifications. She mentioned that she had already suffered six major surgeries in her life, which she associated with a weak quality of her constitution. Mrs. Huang, on the other hand, noted:

My constitution is weaker than others, I was ill a lot while I was young and I usually had a heavy flow of menstrual blood. Indeed, I was weak especially while I was young.

In this case, the heavy flow of menstrual blood was viewed as both a symbol and a result of her weak constitution.

Inability to adjust to unfavorable environments also characterized a weakness of one's constitution. A frequently mentioned complaint was "can stand neither hot nor cold weather". For example, Mr. Lin believed that he had a weak constitution because he possessed a cold constitution and should therefore favor hot condition, but he, unfortunately, was also afraid of hot weather.

The hot/cold dichotomy (see Table 2) is another representation of personal constitutions, mentioned by $23 \%$ of the elders*. A hot constitution was most often characterized by "favoring cold foods" or "feeling more comfortable during the winter". The opposite was true for a cold constitution. As a result, those who claimed a hot constitution always placed restrictions on hot foods, especially tonics. 
A widely shared common sense among these elderly is about hot/cold constitution and the consumption of ginseng. The prevalent belief is that some individuals' constitutions are not compatible with ginseng's medicinal properties. In the lay belief system, two major types of ginseng, Korean and American, can be identified. In the eyes of lay people these two not only look different but also suit the needs of people with different constitutions. Korean ginseng is stronger in its effect and has a "hot" nature. American ginseng, on the other hand, is gentle and "cold" in essence. Basically, respondents who possessed a hot constitution or whose constitution would turn hot on certain occasions should avoid consuming hot tonics like Korean ginseng. Otherwise it will generate inner heat (huo qi), which might cause various disorders. It was the discomfort caused by consuming hot substances or foods that made people come to learn the nature of their constitutions.

Some informants even more specifically identified certain of their organs as hot or cold. For example, some had constitutions with intense liver fire (gan huo wang). Mr. Liau said that he had a "cold stomach" but was "hot in the intestines". He complained that the combination always caused problems in his bowel movements.

For some $(7 \%)$ the quality of hot/cold could also change with situations. For example, one respondent stated that his constitution was basically cold, but when he felt tired it changed from cold to hot.

Constitution was also characterized in terms other than strong/weak and hot/cold (Table 2). These characteristics usually were mentioned in addition to one of the above descriptors. For example, some people related their constitution to their body shape: "a typical fat guy" or "with a skinny, delicate shape". Some mentioned blood type. Others, as in the case of $\mathrm{Mr}$. $\mathrm{Hu}$, related constitution to certain physiological attributes. He regarded his constitution as that of "a typical skinny, low blood pressure type".

Another noticeable designation related to characteristics of personality, personal disposition or capability. Some people were proud of their "good temper", "patience", and "endurance"; others considered themselves "very intelligent".

For some people, constitution is more than strong/weak, hot/cold, fat/skinny, or irritable/calm. It is a constellation of morphological, physical, and mental dispositions. These are the informants who fell into more than one category on Table 2. For example, Mr. Wang thought the constitution he possessed was a combination of "B blood type, prone to cold and weakness, with low blood pressure and low body temperature". A female respondent complained:

I have a bad constitution. I cannot raise heavy stuff. Basically my constitution is cold in nature so I had better never get a cold. Because every time when I got a cold I always had a fever.

A bad constitution is one that is functionally weak and cold. For this elderly woman, these two characteristics were enough to justify her "weak" constitution.

Although these descriptions of constitution reflect perceptions of the body, they are, in and of themselves, devoid of clinical significance. Thus, constitution is viewed as something that may explain aspects of health status, but it also explains other aspects of the individual's life that are not, by Western standards, health related. Among the older Chinese, then, constitution itself is not necessarily an object for medical intervention.

\section{CONSTITUTION AND LIFE EXPERIENCE}

Family heredity was frequently recognized as critical for shaping one's constitution. In a description of his strong constitution, Mr. Chang, a 79year-old retired colonel, particularly stressed his distinguished heritage. "For four generations", he stated proudly, "the men of my family were all military officers". His comment and that of $\mathrm{Mr}$. Yuang (many of his children and grandchildren were Ph.D.s) both suggested the heritability of personal constitution. Mr. Chang represented his constitution as inherited from previous generations and Mr. Yuang thought that his constitutional intelligence had passed to his offspring.

Birth order was also considered relevant. The oldest in his family, Mr. Dong asserted that the first child normally inherited the best from the parents and that being the first child contributed considerably to one's constitution. But another respondent, Mr. Lai noted:

I was born the youngest of seven children. All of the members of my family nurtured me greatly, which, I think, has brought me a strong constitution.

More people, however, emphasized the importance of their parents' health, especially that of their mother. The story provided by $\mathrm{Mr}$. $\mathrm{Wu}$ is an example of this belief:

I was born prematurely. My mother was very weak after she gave birth to me. So I was not breast fed. As a very weak child, I learned how to walk as late as when I was four. My constitution is relatively hot. I loved athletics while I was a junior high student. At that time I won many championships.

\section{Another respondent recalled:}

Basically my constitution is fine, although I used to be a very weak child. My mother had TB when I was born. As a result, I have paid much attention to my health and lifestyle. I am very self-disciplined and have never stayed up late in my life.

Along with the mother's health status is a concern for the mother's age. A respondent noted: 
I am afraid of cold probably because I have been thin all my life. I was a child difficult to raise because my mother was already 45 while she gave birth to me.

Mrs. Chao also referred to her mother's age:

I was born when my parents were old. My mother was already 42 when I was born. Consequently, I was sick a lot during my childhood, which prohibited me from a normal schooling. Only after I went to Manchuria to stay with my older brother did I become stronger. However, although I was constitutionally unhealthy, I paid much attention to recuperation each time after I gave birth. I usually employed supplemental shots during the period of doing the month*. I think that's why I've remained healthy as I got old.

Life experience during the younger years was thought to have a great deal to do with the characteristics of constitution. Some elderly men believed that their military service had helped them build a strong constitution. In describing her hot constitution, Mrs. Huang stated:

I think my constitution is quite good, though I didn't pay too much attention to my health actually. Nevertheless, as a child I was very much nurtured by my parents and my grandparents. You know, girls were especially adorable in Beijing.

A well-fed, easy life was not necessarily appreciated. For example, Mr. Wang complained that he had a poor constitution because:

I was very much spoiled by my family. They wouldn't let me do any work, which deprived me of the opportunity to strengthen my body. As a result, the "basis" of my constitution is quite weak.

The story of 90-year-old Mrs. Wu also is an interesting example:

My constitution has become better since I migrated from the mainland to Taiwan. I was born in a well-to-do family and we used to have servants around. The beginning of life in Taiwan was a nightmare for me. We had left everything behind on the mainland and had become ordinary people and no longer had servants helping out with daily routines and household chores. Since then I learned how to be independent and to do everything by myself. Eventually, this kind of life did greatly strengthen my constitution.

*The "doing the month" addresses the significance of gender and life-cycle in accounts of one's constitution. "Doing the month" (zuo yue zi) is a traditional practice in obstetrical care in China and is still common in contemporary Taiwan. According to this tradition, after giving birth a woman should be confined to home a full month for recuperation. During this period of time, she has to adhere to a regimen of "do's and don'ts" in order to ensure a full convalescence from child birth. The activities under restriction include dietary choices, personal hygiene, physical activities and social interactions (Pillsbury, 1978). By doing well during the month, an originally weak constitution can be strengthened. Many, especially among the older generations, believe that the customary practice of recuperation after child birth has been made an opportunity for Chinese women to build up their constitutions. However, it should be noted that an originally strong constitution could be run down if "doing the month" was not carried out appropriately.
These statements also imply another dimension along which concepts of constitution vary; that is, the extent to which constitution can be changed. Nearly $59 \%$ of the Chinese elderly thought that constitution could change. On the other hand, $28 \%$ thought that constitution could not be changed; $8 \%$ did not know and 5\% did not answer.

Those who believed that constitution could not be changed stated that one's constitution is inborn, invariant, and, at its core, sustained throughout one's lifetime. Thus, constitution is fixed at birth and cannot be changed. As one elder noted, "Constitution can never be changed. However, it is quite possible to change one's health status". Another elder said:

There is little possibility to change one's constitution. Basically, not many differences can be found among personal constitutions. After all, everyone comes to the world through the same process. What can possibly be changed is one's state of mind.

For this group, constitution represents an individual's core trait, inborn and everlasting throughout their life.

However, the majority of the elders believed that constitution varied because of personal and situational factors. An extreme example of this outlook was presented by Mr. Cheng:

One's constitution keeps changing every day. So I also keep warning myself to be cautious of my own health. Other people's conduct presents a mirror to me. For example, every time I saw a person who did not straighten his shoulders and back up I would warn myself never to act like him. I should always keep myself in good posture.

Another respondent agreed, "Constitution can certainly be changed. What cannot be changed is personality".

According to the elders, four major forces may bring changes in the nature of one's constitution. The first is age. Personal constitution can change with the advance of age, and the accompanying changes are most likely negative. Environment is another source of change. Environment here refers broadly to the external environment such as living and working spaces and practices, as well as other natural conditions. The third factor includes serious diseases or major operations, which may change one's constitution temporarily or permanently. The fourth is personal behavior.

The respondents who mentioned personal behaviors cited exercise or other types of physical training, diet, relaxation, and regular habits. "Regulation and nourishment" (tiao yung) in particular was the most frequently stated strategy for changing one's constitution for the better. Many respondents firmly believed that the quality of their constitution could be improved as long as it was regulated and nourished consistently. Nourishment by means of the administration of health foods or herbal tonics along with an emphasis on regularity of daily life could improve the constitution. For 
Table 3. Symptoms, diseases and type of personal constitution

\begin{tabular}{|c|c|c|c|c|c|}
\hline & \multicolumn{3}{|c|}{ strong/weak dimension } & \multicolumn{2}{|c|}{ hot/cold dimension } \\
\hline & $\begin{array}{c}\text { strong } \\
(n=68)(\%)\end{array}$ & $\begin{array}{c}\text { normal } \\
(n=71)(\%)\end{array}$ & $\begin{array}{c}\text { weak } \\
(n=20)(\%)\end{array}$ & $\begin{array}{c}\text { hot } \\
(n=23)(\%)\end{array}$ & $\begin{array}{c}\text { cold } \\
(n=17)(\%)\end{array}$ \\
\hline \multicolumn{6}{|c|}{ Overall indicators } \\
\hline No. of symptom-affected body systems & 1.95 & 2.09 & $2.70^{\mathrm{a}}$ & 2.09 & 2.35 \\
\hline No. of disease-affected body systems & 1.82 & 1.91 & 1.95 & 1.87 & 1.94 \\
\hline \multicolumn{6}{|c|}{ Mean number of symptoms in each system } \\
\hline Central nervous & 1.41 & 1.48 & 1.70 & 1.39 & 1.23 \\
\hline Gastrointestinal & 0.38 & 0.43 & 0.35 & 0.47 & $0.18^{\mathrm{b}}$ \\
\hline Joint & 0.31 & 0.61 & $0.75^{\mathrm{b}}$ & 0.43 & 0.41 \\
\hline Muscular & 0.13 & 0.10 & $0.40^{\mathrm{a}}$ & 0.30 & 0.35 \\
\hline Metabolic & 0.54 & 0.48 & $1.05^{\mathrm{b}}$ & 0.39 & 0.70 \\
\hline \multicolumn{6}{|c|}{ Mean number of diseases in each system } \\
\hline Cardiovascular & 0.65 & 0.64 & 0.50 & 0.60 & 0.53 \\
\hline Respiratory & 0.24 & 0.27 & 0.30 & 0.35 & $0.24^{\mathrm{a}}$ \\
\hline Intestinal & 0.26 & 0.25 & 0.45 & 0.22 & 0.41 \\
\hline Genitourinary & 0.24 & 0.30 & 0.15 & 0.26 & 0.18 \\
\hline Skeletomuscular & 0.38 & 0.51 & 0.50 & 0.48 & 0.47 \\
\hline Metabolic & 0.20 & 0.28 & 0.40 & 0.22 & 0.18 \\
\hline
\end{tabular}

${ }^{\mathrm{a}} p<0.05 ;{ }^{\mathrm{b}} p<0.01$.

example, it was commonly believed that a longterm intake of ginseng could modify one's constitution*. In addition, there were people who believed that state of mind or emotional disturbances, such as anxiety, could alter the nature of the constitution. Hence, maintaining a "balanced" emotional state became another critical issue for the assurance of the quality of one's constitution.

\section{CONSTITUTION AND HEALTH STATUS}

Table 3 presents the mean number of symptoms and diseases reported for major body systems by informants who said their constitutions were strong, normal, or weak, and for informants who said their constitutions were hot or cold. The mean number of conditions reported is greater for those with weak constitutions when compared to those with strong or normal constitutions. This trend is statistically significant in the case of symptoms. When disaggregated by body system, the relationships between symptoms and strong/weak constitution are even more apparent. Those reporting weak constitutions have significantly more symptoms in the joints, muscular, or metabolic body systems than do those reporting strong or normal constitutions. However, this relationship is not observed for the number of diseases in each system.

\footnotetext{
*Ginseng is the most well-known and most widely used hygienic ingredient among Chinese people. About 30\% of the sample regularly used ginseng. It was either consumed alone or in combination with other herbs or medicinal materials, in the form of prescriptions like ginseng tea, ginseng meat soup, or ginseng wine. In any case, the tonics can be considered a type of food supplement. For a brief discussion of ginseng and its effects, especially its pharmacological activities and clinical usefulness, see Chen (1973). The systematic, empirical examination of lay people's belief in the effects of ginseng and its utility, to our knowledge, is not available.
}

Although persons with cold constitutions report slightly more symptoms and diseases than those with hot constitutions, the differences are not statistically significant. Neither group has a clear advantage when symptoms and diseases are examined by body system: People reporting cold constitutions have significantly fewer gastrointestinal symptoms and diseases of the respiratory system than those reporting hot constitutions.

The statistically significant relationships between symptoms and the strong/weak dimension are consistent, and in the expected direction. Diseases do not have a consistent relationship with this dimension. It appears, however, that the relationship between the hot/cold dimension and both symptoms and diseases is slight.

Table 4 shows the relationship between constitution and self-rated health status and satisfaction with health. The relationships between the strong/ weak dimension and both self-report health measures are statistically significant. Those saying their constitutions are weak are least likely to report their health as very good or good. Those saying they have a normal constitution are more likely to rate their health as very good or good than those who say their constitution is strong. The same pattern applies to the question on satisfaction: Those who say their constitution is weak are least likely to be satisfied with their health and those saying their constitution is normal are most likely to be satisfied.

The association of hot/cold constitution with selfrated health status also is statistically significant. The elderly who claim a hot constitution are more likely to consider their health status very good or good than those who claim a cold constitution. Although not statistically significant, the association between hot/cold constitution and health satisfaction follows the same pattern. 
Table 4. Self-rated health and health satisfaction for different types of constitution

\begin{tabular}{|c|c|c|c|c|c|}
\hline & \multicolumn{3}{|c|}{ strong/weak dimension } & \multicolumn{2}{|c|}{ hot/cold dimension } \\
\hline & $\begin{array}{c}\text { strong } \\
(n=68)(\%)\end{array}$ & $\begin{array}{c}\text { normal } \\
(n=71)(\%)\end{array}$ & $\begin{array}{c}\text { weak } \\
(n=20)(\%)\end{array}$ & $\begin{array}{c}\text { hot } \\
(n=23)(\%)\end{array}$ & $\begin{array}{c}\text { cold } \\
(n=17)(\%)\end{array}$ \\
\hline \multicolumn{6}{|c|}{ Self-rated health } \\
\hline Very good/good & 70.2 & 82.1 & $55.0^{\mathrm{b}}$ & 78.3 & $47.6^{\mathrm{a}}$ \\
\hline Fair/poor/very poor & 29.8 & 17.9 & 45.0 & 21.7 & 52.4 \\
\hline \multicolumn{6}{|c|}{ Health satisfaction } \\
\hline Very satisfied/satisfied & 86.7 & 89.5 & $65.0^{\mathrm{a}}$ & 73.9 & 64.7 \\
\hline $\begin{array}{l}\text { Fairly satisfied/dissatisfied/very } \\
\text { dissatisfied }\end{array}$ & 13.3 & 10.5 & 35.0 & 26.1 & 35.3 \\
\hline
\end{tabular}

\section{DISCUSSION}

The comments of these informants indicate that constitution is a recognized and familiar concept in Chinese society, both in Taipei and Los Angeles. It is likely that the residents of both cities share ideas about health that are rooted in their very early experiences. Apparently the subsequent experiences of the Chinese elders in Los Angeles, including their exposure to Western medical care, did not cause them to change their view of the role of constitution in their lives.

To some of them, constitution is a core trait, a constellation of physical and mental representations. Its essential pattern will abide throughout their lives with little variation. To others, constitution is not simply a set of inborn traits. Rather, it is a set of states which can be modified, developed, and strengthened.

The responses of the elderly Chinese indicate that constitution can explain much that they experience both physically and mentally. Thus, the concept of constitution provides a frame of reference for understanding everyday existence.

The comments of the Chinese elderly also indicate that personal constitution is multidimensional. On analysis, these comments provided three types of information about constitution: (1) types of constitutions; (2) qualities of each type; and (3) factors influencing how constitutions develop and, in some cases, change.

The two dimensions of constitution discussed most frequently were the strong/weak dimension and the hot/cold dimension. These are quite different than those used in traditional Chinese medical texts. For example, there were few references either to yin/yang or the five evolutive phases. This suggests that lay constructions of constitution may be rooted in folk traditions that may, to some extent, be independent of traditional Chinese medicine. Whether or not the Chinese elderly indicated that constitution could be changed, nearly all of them explained their constitutions by referring both to life events, including birth order and parents' age at birth, and to family heredity.
Further, a number of the Chinese elderly indicated that they had come to understand their constitutions through their experiences over the life course. Thus, many of them provided a "natural history" of their constitutions. Rather than focusing on specific diseases, they rationalized their assessments of the strength of their constitutions by referring to functional capabilities. Many of them also indicated that their constitutions would be weakened if they did not follow certain "rules". They learned the rules largely from long-term trial and error (see Lee, 1980 for a similar explanation for Hong Kong).

The most distinctive example of this involves the hot/cold dimension of constitution. Most elderly Chinese designated their constitution as hot or cold simply because they would feel uncomfortable or certain symptoms would appear if they were too exposed to certain hot or cold conditions such as weather and/or foods. Thus, the discomfort experienced coincidentally with the consumption of different foods taught people the nature of their constitutions. The example of which type of ginseng people with different constitutions should consume illustrates the point.

The extent to which results showing a significant relationship between constitution and self-reported health status can be interpreted is limited by the small number of persons who reported a weak constitution. Of course, it is difficult to know either the connotative meanings that may have been evoked by the questions on health status or the frames of reference within which the elderly Chinese might have interpreted them. Nonetheless, as the comments reported above indicate, constitution is a concept that organizes the experiences of the body in ways that are both quite different from and more inclusive than Western concepts of health. That there are significant relationships between constitution (especially strong/weak) and some indicators of health status is not surprising, since constitutions are identified and understood, in part, according to ongoing health experiences. That these relationships are not strong enough to suggest that the Western concept of health status and the Chinese concept of 
constitution are equivalent, also is not surprising, since one of the benefits of understanding one's constitution is the resulting ability to act in ways to prevent probable negative outcomes.

Regarding the interpretation of constitution and health status as competing explanations for health experiences, the conclusion from Lee (1980: 368) about perceptions and uses of Chinese and Western medicine in Hong Kong is instructive. He notes that

deep-rooted in Chinese culture are two general practical principles of everyday life: (1) every approach is bound to have its own strengths and weaknesses, and (2) a person should adopt the strengths of one approach to complement the weaknesses of another approach.

It seems that elders in both Taipei and Los Angeles have adopted what they perceive to be the strengths of Western ideas about health while retaining what they perceive to be the strengths of their Chinese tradition. The two are not incompatible. Rather than suggest that the elders in Los Angeles remain untouched by Western ideas, it might be more accurate to suggest that elders in Taipei are similarly touched by Western ideas, that is to say, only partially.

\section{CONCLUSION}

Although constitution is a common construct in Chinese daily life, there are few data on the range of contexts in which it is invoked. However, traditional Chinese medicine recognizes constitution as a specific etiologic factor in many diseases. Among lay Chinese, it appears that constitution is used as a way of making sense of ambiguous conditions. In situations where the etiology of a perceived health problem is not clear, constitution becomes the obvious non-supernatural explanation. Sankar (1984) makes a similar point about the use of "old age" in the Western medical tradition. This raises the question of what people who believe constitution is unchangeable do about conditions they believe are caused by it.

Whether or not they think it can be changed, however, the Chinese elderly view constitution as a long term, pervasive characteristic that is central to their sense of self. Health status, at least as it is understood in the West, is a more temporal, fluctuating state that would be expected to vary with their experience of illness. The longitudinal data that would be required to determine the extent to which perceived health status varies with illness experience among the informants is not available. However, there are a number of persons in this study reporting either strong constitutions but poor health status or weak constitutions but good health status. It is unlikely that such persons would consider their responses incongruent.
Acknowledgements - Support for this study was partially provided by the CI-79-49 grant from the Chin-Lin Medical Foundation at the Chin-Lin Geriatrics Research Center, Taipei, Taiwan, Republic of China. We wish to thank Dr Virginia Li, Dr Francesca Bray, and Dr Peter Lachenbruch for their comments on the earlier versions of this paper. We also thank Dr Heng-Hung Chang at the Hospital of Chinese Medical College, Taichung, Taiwan for his information concerning personal constitution in Chinese medicine and his specific comments on the results of this study. Correspondence may be addressed to the first author at the School of Public Health, National Taiwan University. No. 1, Section 1, Jen-Ai Rd. Taipei, Taiwan, Republic of China.

\section{REFERENCES}

Bergner, M. (1987) Health status measures: An overview and guide for selection. Annual Review of Public Health 8, 191-210.

Chen, J. Y. (1973) Chinese health foods and herb tonics. American Journal of Chinese Medicine 1(2), 225-247.

Foster, G. (1987) On the origin of humoral medicine in Latin America. Medical Anthropology Quarterly 1(4), 355-393.

Furth, C. and Ch'en, S. Y. (1992) Chinese medicine and the anthropology of menstruation in contemporary Taiwan. Medical Anthropology Quarterly 6(1), 27-48.

Gephart, R. P. Jr. (1988) Ethnostatistics: qualitative foundations for quantitative research. Sage Publications, Newbury Park, CA.

Lee, R. P. L. (1980) Perceptions and uses of Chinese medicine among the Chinese in Hong Kong. Culture, Medicine and Psychiatry 4, 345-375.

Li, X. M. (1981) Nei Ching qi zhi xue shuo chu tian (A preliminary inquiry into the theory of disposition in Nei Ching). Zhe jiang zhong yi zha zhi (Zhe Jing Chinese Medical Journal) 6, 248-250.

Lu, Z. P. (1982) Shi lun nei ching de ti zhi xue shuo (A remark on the physical constitution in Nei Ching). Zhe Jiang Medical Journal 3, 112-113.

Mu, G. C. (1983) Zhong yi ti zhi xue shuo ji qi yi hua (Theory of physical constitution and its ramification in Chinese medicine). Xin zhong yi (New Chinese Medicine) 9, 1-7.

Pillsbury, B. L. K. (1978) "Doing the month": confinement and convalescence of Chinese women after childbirth. Social Science and Medicine 12, 11-22.

Porkert, M. (1977) Intellectual and social impulses behind the evolution of traditional Chinese medicine. In Asian Medical systems: a comparative study, ed. C. Leslie, pp. 63-81. University of California Press, Berkeley, CA.

Sankar, A. (1984) "It's just old age": old age as diagnosis in American and Chinese medicine. In Age and Anthropological Theory, ed. D. Kertzer and J. Keith, pp. 250-280. Cornell University Press, Ithaca, NY.

Shi, Y. (1984) Guan yu ti zhi xue shuo de tao lun (On the theory of physical constitution). Shan dong zhong yi xue yuan xue bao (Journal of Shan Dun Chinese Medical College) 4, 36-39.

$\mathrm{Su}$, Y. C. (1992) The study of the physical constitution of systemic lupus erythematosus. Masters thesis, Graduate Institute of Chinese Medical Science, China Medical College, Taiwan, ROC.

Tian, C. A. (1984) Zhong yi ti zhi xue shuo yu xian dai mian yi li lun (Theory of physical constitution in Chinese medicine and contemporary immunology). Shan dun zhong yi xue bao (Journal of Shan Dun Chinese Medical College) 8(1), 7-10.

Wang, D. P. (1989) Qian yi zhong yi ti zhi xue shuo ji qi lin chuang yi yi (Physical constitution in Chinese medi- 
cine and its clinical implications). Yun Nan zhong yi zha zhi (Yun Nan Chinese Medical Journal) 6, 10-12.

Zhang, W. R. (1992) Shi wu dui ti zhi xing cheng ying xiang de sheng hua yan jiu (Biochemical investigation on foods and the construction of physical constitution). Zhong yi zha zhi (Chinese Medical Journal) 33(7), 4950 .
Zhou, G. X. (1987) Guang dong han zu jian kang ren de zhong yi ti zhi lei xing yu HLA ji yin pin lu fen bu de guan xi (Type of physical constitution and the distribution of HLA gene among healthy people of Han ethnic group in Guan Dong Province). Zhong xi yi jie he zha zhi (Journal of Integrated Chinese and Western Medicine) 9, 519-522. 